\title{
Akkermansia muciniphila improves metabolic profiles by reducing inflammation in chow diet-fed mice
}

\author{
Shaoqian Zhao1,*, Wen Liu1,*, Jiqiu Wang1,*, Juan Shi', Yingkai Sun1, Weiqing Wang1, \\ Guang Ning ${ }^{1,2}$, Ruixin Liu' ${ }^{1}$ and Jie Hong' ${ }^{1}$
}

1Department of Endocrinology and Metabolism, Shanghai Clinical Center for Endocrine and Metabolic Diseases, Shanghai Institute of Endocrine and Metabolic Diseases, China National Research Center for Metabolic Diseases, Ruijin Hospital, Shanghai Jiao Tong University School of Medicine, Shanghai, China 2Laboratory of Endocrinology and Metabolism, Institute of Health Sciences, Shanghai Institutes for Biological Sciences (SIBS), Chinese Academy of Sciences (CAS) \& Shanghai Jiao Tong University School of Medicine (SJTUSM), Shanghai, China

*(S Zhao, W Liu and J Wang contributed equally to this work)

Correspondence should be addressed to R Liu or J Hong Email xiner198287@163.com or hongjie@medmail.com.cn

\begin{abstract}
Abnormal shifts in the composition of gut microbiota contribute to the pathogenesis of metabolic diseases, including obesity and type 2 diabetes (T2DM). The crosstalk between gut microbes and the host affects the inflammatory status and glucose tolerance of the individuals, but the underlying mechanisms have not been elucidated completely. In this study, we treated the lean chow diet-fed mice with Akkermansia muciniphila, which is thought to be inversely correlated with inflammation status and body weight in rodents and humans, and we found that $A$. muciniphila supplementation by daily gavage for five weeks significantly alleviated body weight gain and reduced fat mass. Glucose tolerance and insulin sensitivity were also improved by $A$. muciniphila supplementation compared with the vehicle. Furthermore, A. muciniphila supplementation reduced gene expression related to fatty acid synthesis and transport in liver and muscle; meanwhile, endoplasmic reticulum (ER) stress in liver and muscle was also alleviated by $A$. muciniphila. More importantly, A. muciniphila supplementation reduced chronic low-grade inflammation, as reflected by decreased plasma levels of lipopolysaccharide (LPS)-binding protein (LBP) and leptin, as well as inactivated LPS/LBP downstream signaling (e.g. decreased phosphoJNK and increased IKBA expression) in liver and muscle. Moreover, metabolomics profiling in plasma also revealed an increase in anti-inflammatory factors such as $\alpha$-tocopherol, $\beta$-sitosterol and a decrease of representative amino acids. In summary, our study demonstrated that $A$. muciniphila supplementation relieved metabolic inflammation, providing underlying mechanisms for the interaction of $A$. muciniphila and host health, pointing to possibilities for metabolic benefits using specific probiotics supplementation in metabolic healthy individuals.
\end{abstract}

\author{
Key Words \\ - Akkermansia muciniphila \\ - glucose tolerance \\ - ER stress \\ - inflammation \\ - LBP
}

\section{Introduction}

Metabolic diseases such as obesity and type 2 diabetes (T2DM) have increasing prevalence worldwide because of the sedentary lifestyles and easy access to energy-rich food (Hossain et al. 2007). Abundant evidence shows that the development of metabolic diseases is affected by genetics and environmental factors (Ussar et al. 2015).

Published by Bioscientifica Ltd. 
Gut microbiota, acting as an environmental factor, is linked to the development and progression of obesity and T2DM (Cani et al. 2012). A metagenome-wide association study of gut microbiota in patients with T2DM depicts intestinal dysbiosis and altered composition of gut microbiota (Qin et al. 2012). Chatelier and coworkers revealed that metabolic disorders are associated with a low gene richness in the microbiome (Le Chatelier et al. 2013). Many studies demonstrated that gut microbiota maintain energy homeostasis by affecting gut barrier, which is important for metabolic endotoxemia and metabolic disorders (Cani et al. 2009). These findings raise the possibility of targeting gut microbiota to prevent metabolic diseases and benefit human health.

Accumulating data uncovered the effects of probiotics or prebiotics on human health, providing a new strategy for treatment of metabolic diseases (Cano et al. 2013, Anhe et al. 2015). Meanwhile, several lines of evidence pointed to $A$. muciniphila as a potential probiotic. A. muciniphila is a mucin degrader residing in the mucus layer of the human intestinal tract (Derrien et al. 2008) and maintains the gut barrier (Reunanen et al. 2015). A. muciniphila is established during the first month of the whole life and develops within a year to a level close to that in healthy adults (Collado et al. 2007), accounting for $1-4 \%$ of the bacterial population in the colon of healthy subjects (Belzer \& de Vos 2012). It is capable of using mucin as a carbon, energy and nitrogen source and is able to release sulfate in a free form from mucin fermentation (Derrien et al. 2004). Human and mouse studies repeatedly demonstrate that the abundance of A. muciniphila is reduced in subjects who are overweight or obese (Le Chatelier et al. 2013), as well as in patients with type 1 diabetes (Hansen et al. 2012), except one report showing that $A$. muciniphila is increased in type 2 diabetic subjects (Qin et al. 2012). Based on a recent research, anti-diabetic metformin treatment improved glucose homeostasis in association with increased Akkermansia spp. population in the gut microbiome of mice (Shin et al. 2014). Additionally, it is reported that the integrity of the developing healthy intestinal tract is correlated with an increasing abundance of A. muciniphila (Collado et al. 2007). A study of rodent models manifested that $A$. muciniphila supplementation contributes to the maintenance of the integrity of gut epithelium and helps to improve metabolic disorders in diet-induced obese mice (Everard et al. 2013). These findings together supported that A. muciniphila may have beneficial effects on human health especially in the disease models; however, whether it could also benefit health in normal subjects is still unclear, as are the detailed mechanisms.

In this study, we treated chow diet-fed mice with A. muciniphila at $2 \times 10^{8}$ colony-forming units (CFUs) $/ 200 \mu \mathrm{L}$ by daily gavage for five weeks to reveal the effects of $A$. muciniphila supplementation in normal mice regarding glucose metabolism and inflammation. Our results demonstrated improved adiposity, glucose tolerance and low-grade inflammation in chow diet-fed mice by $A$. muciniphila. Hence, our research provided new evidence for the metabolic benefits of $A$. muciniphila on chow diet-fed mice, pointing out the possible mechanisms underlying the interaction of A. muciniphila and the host's metabolism.

\section{Materials and methods}

\section{Preparation of $A$. muciniphila}

A. muciniphila (ATCC BAA-835) was cultured in sterilized brain heart infusion broth (BD Difco) at $37^{\circ} \mathrm{C}$ in an airtight pot with AnaeroPack (Thermo Fisher Scientific) for approximately $48 \mathrm{~h}$ to reach a late exponential growth phase under strict anaerobic conditions. Cultures were centrifuged at $11,500 \boldsymbol{g}$ for $10 \mathrm{~min}$, washed with sterile PBS for twice, and then the bacterial cells were re-suspended with sterile PBS to $10^{8}$ colony-forming units (CFUs) $/ 200 \mu \mathrm{L}$. It was placed on ice immediately before administering to each mouse by gavage.

\section{Experimental animal}

Six-week-old male specific-pathogen-free (SPF)-grade C57BL/6 mice $(n=20)$ purchased from Shanghai Laboratory Animal Center were maintained in groups of no more than 5 mice per cage with a stable controlled environment and free access to food and water. The mice were raised for 2 weeks without any intervention to adapt to the new environment and then divided into two groups randomly: (1) a normal chow diet (NCD) with sterile PBS (Gibco, Life Technologies) and (2) a NCD with A. muciniphila supplementation. The $A$. muciniphila-treated mice were administered daily with $A$. muciniphila (ATCC BAA-835) by oral gavage at a dose of $2 \times 10^{8} \mathrm{CFUs} / 200 \mu \mathrm{L}$, whereas mice from the first group received sterile PBS as vehicle by daily gavage. The body weight of each mouse was measured once a week during the animal trial, which lasted for 5 weeks. The following experiments were based on these mice except the measurement of food intake and fecal triglyceride content. For measurement of these two

Published by Bioscientifica Ltd 
parameters, we conducted a new gavage experiment in which 40 mice were maintained in 20 cages with 2 mice in each cage and divided into two groups as mentioned previously. The measurements of food intake and energy excretion (fecal triglyceride content) were conducted and recorded with each cage as a unit ( $n=10$ for each group). The measurement of food intake and the collection of stool were conducted in the second week of this trial. All these procedures were approved by the Animal Care Committee of Shanghai Jiao Tong University School of Medicine.

\section{Intraperitoneal glucose tolerance test}

After $14 \mathrm{~h}$ of fasting, the basal blood glucose level of each mouse was measured by the blood taken from the tail vein and recorded in $\mathrm{mmol} / \mathrm{L}$. After an intraperitoneal injection with glucose at a dose of $2 \mathrm{~g} / \mathrm{kg}$ body weight, blood glucose concentrations were measured at 15, 30, 60, 90 and 120 min as described previously (Wang et al. 2013).

\section{Body composition}

The body composition was assessed by an animal whole body composition analyzer (EchoMRI 100H) in accordance with the manufacturer's instructions.

\section{Blood and tissue sample collection}

At the end of the trial, the mice were anesthetized with $10 \%$ chloral hydrate, and the blood from the orbital plexus was collected in tubes. Blood samples were centrifuged for $15 \mathrm{~min}$ at $1000 \mathrm{~g}, 4^{\circ} \mathrm{C}$, and plasma was isolated and stored at $-80^{\circ} \mathrm{C}$ for subsequent biochemical testing, and plasma LBP (Abnova, KA4302) and leptin (Millipore, EZML$82 \mathrm{~K})$ levels were measured using a commercial ELISA kit. Inguinal fat, epididymal fat and the liver and intestine of each mouse were precisely dissected, and immediately stored at $-80^{\circ} \mathrm{C}$ for further analysis.

\section{Triglyceride measurement}

Fecal triglyceride levels were extracted and measured following the manufacturer's instructions using a colorimetric triglyceride assay kit (Bio vision). Briefly, $0.1 \mathrm{~g}$ of feces was homogenized in $1 \mathrm{~mL}$ solution containing $5 \%$ NP-40 in water, it was slowly heated to $80-100^{\circ} \mathrm{C}$ in a water bath for $5 \mathrm{~min}$, and then cooled down to room temperature. The procedure of heating was repeated one more time to solubilize all triglycerides, and then the insoluble material was pelleted by centrifugation $(16,200 \boldsymbol{g}$ for $2 \mathrm{~min})$. An aliquot of the supernatant was collected for subsequent triglyceride level analysis. $50 \mu \mathrm{L}$ test samples and triglyceride standard were added to a 96-well plate. $2 \mu \mathrm{L}$ of lipase was added to each standard, and sample well, samples and lipase were mixed and incubated $20 \mathrm{~min}$ at room temperature to convert the triglyceride to glycerol and fatty acid. $50 \mu \mathrm{L}$ of the reaction mix containing triglyceride probe and triglyceride enzyme were mixed and added to each well, incubating at room temperature for $60 \mathrm{~min}$. The absorbance at $570 \mathrm{~nm}$ was measured, and the TG standard curve was plotted to calculate the concentration of triglyceride. For plasma triglyceride measurement, we used a triglyceride assay kit (Kehua Bio-Engineering, Shanghai, China) according to its instructions. In brief, mixed reagents were added to both the plasma sample and the standard sample, incubating in $37^{\circ} \mathrm{C}$ for $5 \mathrm{~min}$, and then the absorbance at $550 \mathrm{~nm}$ was measured and the TG standard curve was plotted to calculate the concentration of triglyceride in plasma.

\section{Oil Red O staining}

Liver and muscle were dissected from each mouse $(n=9$ and 8 mice for each group, respectively) and fixed with $4 \%$ paraformaldehyde for more than $24 \mathrm{~h}$, and then embedded in OCT. The tissue was sectioned, and the thickness was approximately $8-10 \mu \mathrm{m}$. Before Oil Red O staining, slides were stored at $-20^{\circ} \mathrm{C}$. Slides were dried for 10 min, washed three times with PBS and stained with Oil Red $\mathrm{O}$ solution for $15 \mathrm{~min}$. Rinse the slides for $1 \mathrm{~min}$ with water, and the nuclei were counter-stained by immersion for 1 min in hematoxylin solution, and then washed by flow water. After covering the slide, three fields of each mouse were photographed and analyzed.

\section{RNA isolation and real-time polymerase chain reaction}

Total RNA was extracted from tissues using TRIzol Reagent (Invitrogen), and $1 \mu \mathrm{g}$ of RNA was transcribed following the manufacturer's instruction by using the Reverse Transcription System Kit (Promega), which produced high-quality complementary DNA. A real-time polymerase chain reaction was performed on LightCyclerH 480 (Roche) using SYBR Green II Master (Takara). All mRNA quantification data were normalized to the housekeeping 36B4 gene. All samples were run in duplicate in a single 384-well reaction plate, and data of the reaction were http://jme.endocrinology-journals.org DOI: 10.1530/JME-16-0054
() 2017 Society for Endocrinology Printed in Great Britain
Published by Bioscientifica Ltd 
collected and analyzed according to the $\Delta \Delta C_{\mathrm{T}}$ method. Primers used in this study are listed in Supplementary Table 1 , see section on supplementary data given at the end of this article.

\section{Protein preparation and western blotting}

Proteins were extracted from dissected tissues and prepared using radioimmunoprecipitation assay buffer. All these samples were from different mice of the two groups and subjected to concentration determination and immunoblot assay with the indicated antibodies. Primary antibodies phospho-Ser ${ }^{473}$ AKT (\#4060), AKT (\#9272), phospho-Ser ${ }^{51}$ EIF2A (\#9721), phospho-PERK Thr980 (\#3179), phospho-SAPK/JNK Thr183/Tyr185 (\#9251), JNK (\#9252) and IKBA (\#4814) were obtained from Cell Signaling Technology (CST) to detect the targeted proteins. Heat Shock Protein90 (Hsp90, \#4874) was purchased from Cell Signaling Technology (CST), and used as the internal control. The blotting bands were visualized using Image Quant LAS 4000 following the manufacturer's guide.

\section{Statistical analysis}

Significant differences between the two groups were assessed by unpaired two-tailed Student's $t$-test. All of these data were tested for the normality before using Student's $t$ test. For non-normal distributions, nonparametric statistical analysis such as Mann-Whitney $U$ test was used. Numerical data are expressed as mean \pm S.D. In these figures, data with '*' are significantly different at $P<0.05$, whereas data with '**' indicate a meaningful difference at $P<0.01$. All the results were considered statistically significant when $P<0.05$.

\section{Metabolites extraction and sample derivatization}

A sample of $20 \mu \mathrm{L}$ of thawed plasma sample and $80 \mu \mathrm{L}$ of cold methanol was added successively into an Eppendorf tube, successively. The mixture was vortex-mixed for $30 \mathrm{~s}$, and placed at $-20^{\circ} \mathrm{C}$ for $1 \mathrm{~h}$ prior to centrifugation at $16,000 \mathrm{~g}$ and $4^{\circ} \mathrm{C}$ for $15 \mathrm{~min}$. In total, $70 \mu \mathrm{L}$ of supernatant were transferred into a glass vial containing $10 \mu \mathrm{L}$ of $0.05 \mathrm{mg} / \mathrm{mL}$ dulcitol and $0.02 \mathrm{mg} / \mathrm{mL}$ of L-phenylalanine$13 \mathrm{C} 9,15 \mathrm{~N}$ as the internal standard. The mixture was dried under a gentle nitrogen stream. The glass vial with dry residue was added with $30 \mu \mathrm{L}$ of $20 \mathrm{mg} / \mathrm{mL}$ methoxylamine hydrochloride in anhydrous pyridine. The resultant mixture was vortex-mixed vigorously for $30 \mathrm{~s}$ and incubated at $37^{\circ} \mathrm{C}$ for $90 \mathrm{~min}$. A $30 \mu \mathrm{L}$ of BSTFA (with $1 \%$ TMCS) was added into the mixture and derivatized at $70^{\circ} \mathrm{C}$ for $60 \mathrm{~min}$.

\section{Gas chromatography-mass spectrometry analysis}

Metabolomics instrumental analysis was performed on an Agilent 7890A gas chromatography system coupled to an Agilent 5975C inert MSD system (Agilent Technologies). A HP-5ms fused-silica capillary column $(30 \mathrm{~m} \times 0.25 \mathrm{~mm} \times 0.25 \mu \mathrm{m}$; Agilent J\&W Scientific, Folsom, CA, USA) was used to separate the derivatives. Helium (>99.999\%) was used as a carrier gas at a constant flow rate of $1 \mathrm{~mL} / \mathrm{min}$ through the column. Injection volume was $1 \mu \mathrm{L}$ by splitless mode, and the solvent delay time was $6 \mathrm{~min}$. The initial oven temperature was held at $70^{\circ} \mathrm{C}$ for $2 \mathrm{~min}$, ramped to $160^{\circ} \mathrm{C}$ at a rate of $6^{\circ} \mathrm{C} / \mathrm{min}$, to $240^{\circ} \mathrm{C}$ at a rate of $10^{\circ} \mathrm{C} / \mathrm{min}$, to $300^{\circ} \mathrm{C}$ at a rate of $20^{\circ} \mathrm{C} / \mathrm{min}$, and finally held at $300^{\circ} \mathrm{C}$ for $6 \mathrm{~min}$. The temperatures of the injector, transfer line and electron impact ion source were set to $250^{\circ} \mathrm{C}, 290^{\circ} \mathrm{C}$ and $230^{\circ} \mathrm{C}$, respectively. The electron energy was $70 \mathrm{eV}$, and data were collected in a full scan mode $(\mathrm{m} / \mathrm{z}$ 50-600).

\section{Data preprocessing and statistical analysis}

The extraction, alignment, deconvolution and further processing of raw gas chromatography-mass spectrometry (GC-MS) data were referred to the previous published protocols (Gao et al. 2010). The mass window was set to $80-600 \mathrm{~m} / \mathrm{z}$. The final data were exported as a peak table file, including observations (sample name) and variables (rt_mz). The data were normalized against internal standard dulcitol before performing univariate and multivariate statistics.

The peak table (named matrix $\mathrm{X}$ ) file was imported to Simca-P (version 11.0, Umetrics AB, Umeå, Sweden), where multivariate statistical analysis, such as PCA, PLS-DA and OPLS-DA were performed. All data were mean-centered, and unit variance (UV) scaled prior to multivariate statistical analysis. The quality of the models is described by the R2X or R2Y and Q2 values. R2X (PCA) or R2Y (PLS-DA and OPLS-DA) is defined as the proportion of variance in the data explained by the models and indicates the goodness of fit. Q2 is defined as the proportion of variance in the data predictable by the model and indicates the predictability of the current model, calculated by cross-validation procedure. To avoid model overfitting, a default 7-round cross-validation

Published by Bioscientifica Ltd. 
in Simca-P was performed throughout to determine the optimal number of principal components. The values of $\mathrm{R} 2 \mathrm{X}, \mathrm{R} 2 \mathrm{Y}$ and $\mathrm{Q} 2$ were used as indicatives to assess the robustness of a pattern-recognition model.

\section{Identification and structural validation of differential metabolites}

The differential metabolites were determined by the combination of the variable importance in the projection (VIP) value $(>1)$ of OPLS-DA model and the $P$ values $(<0.05)$ from two-tailed Student's $t$ test on the normalized peak intensities. Fold change was calculated as the binary logarithm of average normalized peak intensity ratio between Group 1 and Group 2, where the positive value means that the average mass response of Group 1 is higher than that of Group 2. The structural identification of differential metabolites was performed as follows. The AMDIS software was applied to deconvolute mass spectra from raw GC-MS data, and the purified mass spectra were automatically matched with an in-house standard library including retention time and mass spectra, Golm Metabolome Database and Agilent Fiehn GC/MS Metabolomics RTL Library.

\section{Results}

\section{Effects of A. muciniphila supplementation on body weight gain, fat mass, glucose tolerance and insulin signaling in metabolic tissues}

In context of the comparable baseline body weight (Supplementary Fig. 1A), supplementation of A. muciniphila at a dose of $10^{8} \mathrm{CFUs} / 200 \mu \mathrm{L}$ for five weeks alleviated body weight gain (Fig. 1A) and significantly reduced fat mass as well as increased lean mass in mice fed a normal chow diet (Fig. 1B) compared with the vehicle-administered control mice. Moreover, the visceral fat weight that was more linked with the pathogenesis of insulin resistance was decreased more obviously (Fig. 1C). Food intake and fecal triglyceride content were unaltered by $A$. muciniphila supplementation (Supplementary Fig. 1B and C). Plasma triglyceride levels were unaltered (Fig. 1D). Notably, although the fasting glucose level was not changed by A. muciniphila (Fig. 1E), which was in agreement with a previous study (Everard et al. 2013), we found that glucose tolerance was greatly improved by A. muciniphila supplementation in mice fed chow diet, as reflected by intraperitoneal glucose tolerance test (IPGTT) (Fig. 1F) and the corresponding reduced area under curve (AUC) (Fig. 1G). Although fasting plasma insulin levels were comparable between the two groups (Fig. 1H), we detected a significant increase in phosphoAKT Ser473 levels in liver (Fig. 1I) and muscle (Fig. 1J) in $A$. muciniphila group, suggesting an improved insulin sensitivity in liver and muscle. As insulin inhibits the transcriptional expression level of key gluconeogenetic enzymes, phosphoenolpyruvate carboxykinase (PEPCK) and glucose-6-phosphatase (G6P) (Lochhead et al. 2000), increased insulin sensitivity in the liver consequently resulted in decreased hepatic G6P and Pepck expression (Fig. 1K). These results together demonstrated that A. muciniphila supplementation improved energy homeostasis and glucose tolerance in chow diet-fed mice.

\section{A. muciniphila supplementation improves lipid accumulation in liver and muscle}

Given the improved fat mass and glucose tolerance, we next examined lipid metabolism in insulin-responsive tissues. Interestingly, A. muciniphila supplementation significantly decreased the gene expression of sterol regulatory elementbinding proteins (SREBP1c), fatty acid translocase (CD36) involved in lipogenesis and the transport of fatty acids both in liver (Fig. 2A) and in muscle (Fig. 2B). SREBP1c encodes a transcription factor that binds to the sterol regulatory element-1 (SRE1), which is a decamer flanking the low-density lipoprotein receptor gene and some genes involved in sterol biosynthesis (Capel et al. 2013, Kim et al. 2015). CD36 contributes to fatty acids translocation (Wilson et al. 2016). Meanwhile, the expression of acetylCoA carboxylase (ACC) was significantly downregulated by A. muciniphila supplementation in muscle (Fig. 2B). The most important function of ACC is to provide the malonyl-CoA substrate for the biosynthesis of fatty acids (Tong 2005). However, we did not observe similar changes in adipose tissue (Fig. 2C). Gene expression of C/EBPA and PPARG involved in adipocyte differentiation as well as in body weight homeostasis was either unchanged in adipose tissues (Supplementary Fig. 2) (Lefterova et al. 2008). Oil Red O staining was further used for fat deposition measurement showing reduced fat accumulation in A. muciniphila supplemented group than the control group both in liver and in muscle (Fig. 2D and E). The results showed that $A$. muciniphila supplementation strikingly attenuated fat deposition in hepatic tissue and muscle, suggesting that $A$. muciniphila supplementation may reduce lipid accumulation in liver and muscle thereby improving insulin sensitivity. 
A

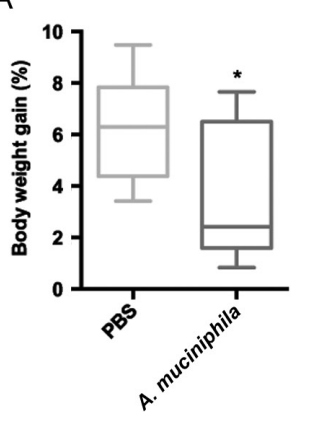

B

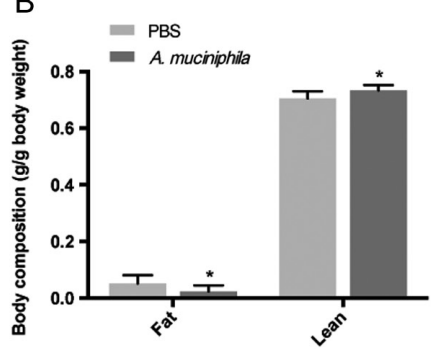

C

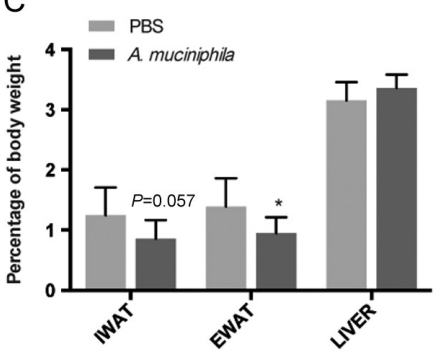

D

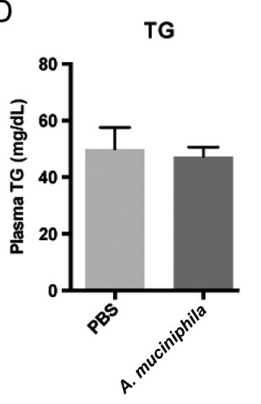

I
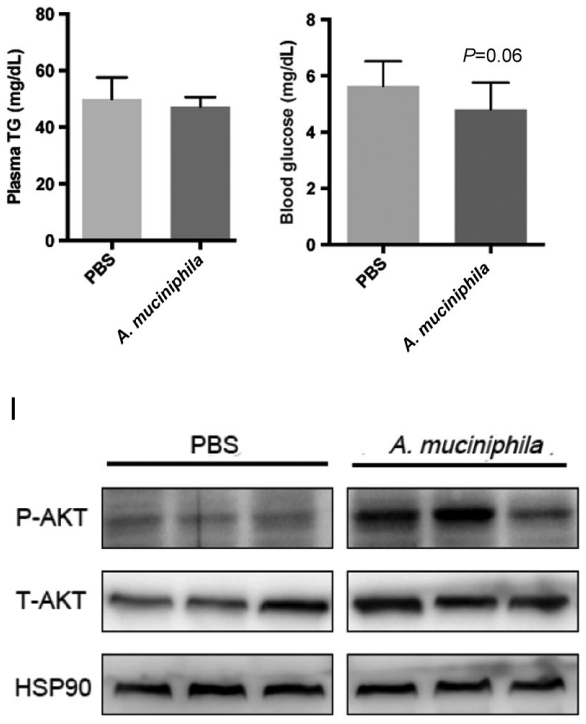

F

A. muciniphila

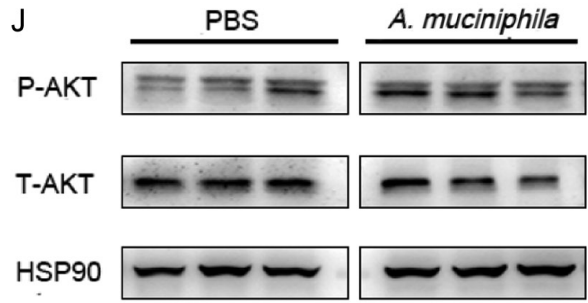

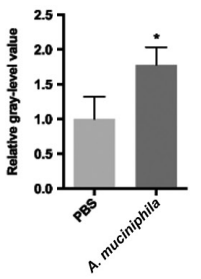

F IPGTT
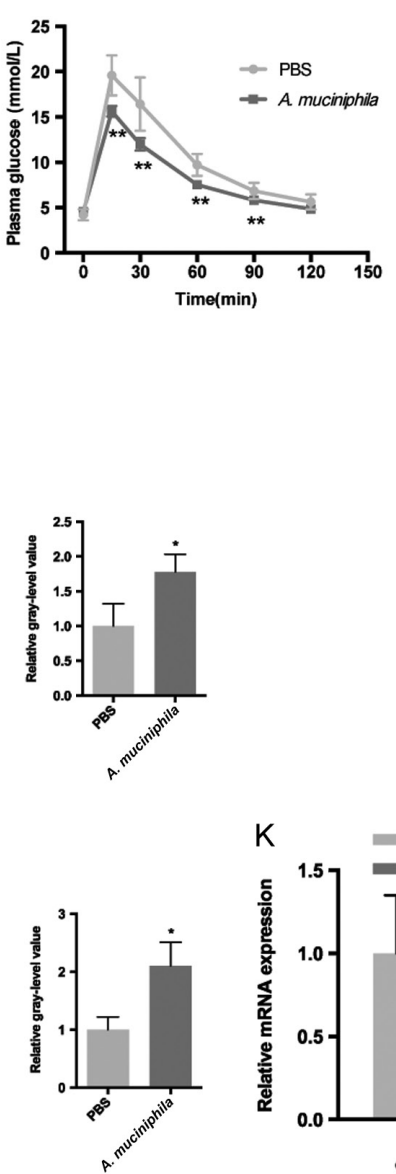

G
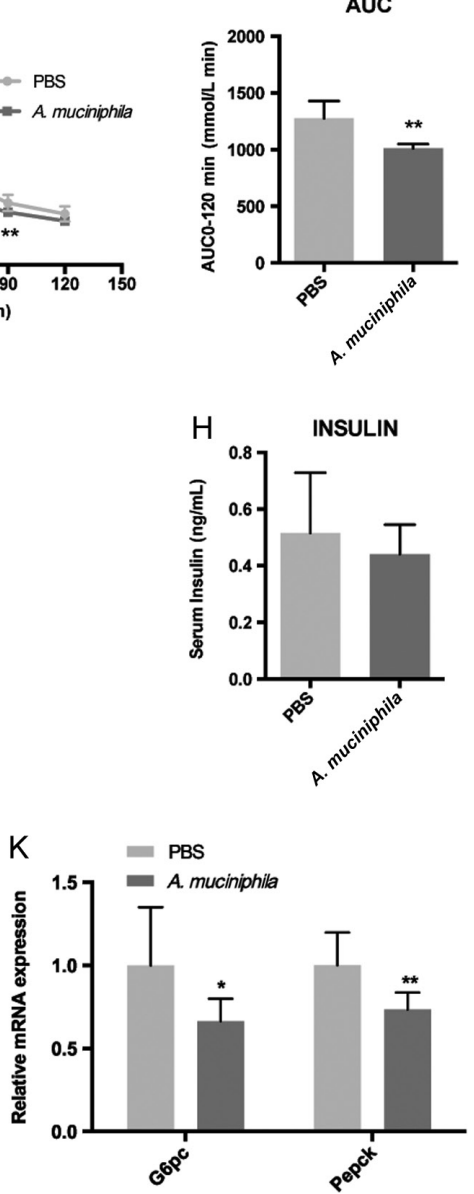

Figure 1

A. muciniphila supplementation alleviated body weight gain, reduced fat mass, improved glucose homeostasis. (A) Average body weight gain (\%) of A. muciniphila-supplemented mice and their counterparts after five-week treatment ( $n=9-8$ for each group, data were shown as median \pm interquartile range, Mann-Whitney test, $\left.{ }^{*} P<0.05,{ }^{*} P<0.01\right)$. (B) Total fat mass and lean mass measured using Echo MRI $(n=10-8$ for each group, data shown as mean \pm S.D., unpaired two-tailed $t$-test, $* P<0.05, * * P<0.01$ ). (C) Weight of IWAT, EWAT and LIVER normalized to body weight in $A$. muciniphila-supplemented mice and their counterparts ( $n=10-8$ for each group, data were shown as mean \pm S.D., unpaired two-tailed $t$-test, $\left.{ }^{*} P<0.05, * * P<0.01\right)$. (D) Plasma TG level between

A. muciniphila-treated mice and their counterparts ( $n=9-8$ for each group, data were shown as mean \pm s.D., unpaired two-tailed $t$-test, $* P<0.05, * * P<0.01$ ).

(E) Fasting blood glucose between two groups ( $n=9-8$ for each group, data were shown as mean \pm S.D., unpaired two-tailed $t$-test, $* P<0.05, * * P<0.01$ ).

(F) Glucose tolerance test between two groups ( $n=9-8$ for each group, data were shown as mean \pm s.D., unpaired two-tailed $t$-test, $* P<0.05, * * P<0.01)$. (G) Mean area under the curve (AUC) measured between 0 and $120 \mathrm{~min}$ after glucose injection. (H) Plasma insulin level between $A$. muciniphila-treated mice and their counterparts ( $n=9-8$ for each group, data were shown as mean \pm s.D., unpaired two-tailed $t$-test, $* P<0.05, * * P<0.01$ ). (I and $\mathrm{J}$ ) Protein levels of insulin signaling in liver (I) and muscle (J) of PBS-treated vehicle and A. muciniphila-supplemented mice. The levels of phospho-Akt were normalized to the levels of total Akt. HSP90 was used as the loading control. Quantitation of p-AKT was performed, all these samples were from 3 different mice from each of the two groups. (K) Relative mRNA levels of G6PC and PEPCK in liver ( $n=9-8$ for each group, data were shown as mean \pm s.D., unpaired two-tailed $t$-test, $* P<0.05, * * P<0.01)$.

http://jme.endocrinology-journals.org DOI: 10.1530/JME-16-0054
() 2017 Society for Endocrinology Printed in Great Britain
Published by Bioscientifica Ltd 
A

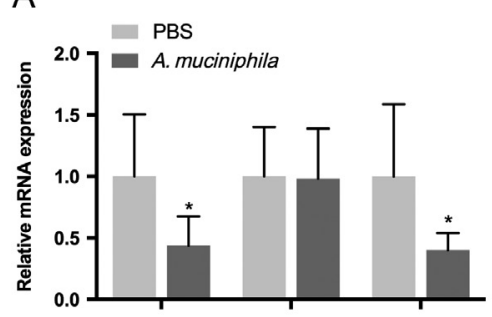

$\mathrm{B}$

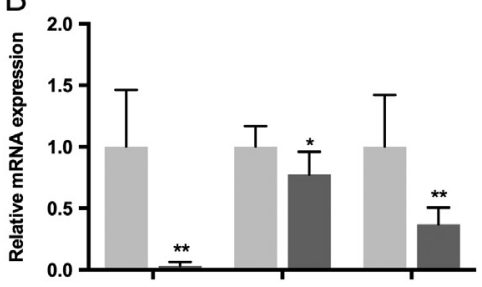

C

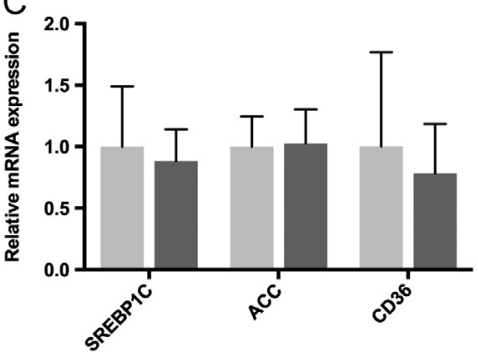

D
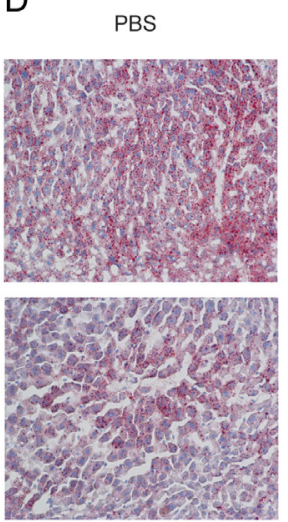

E

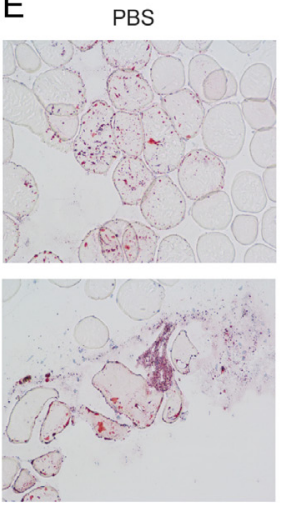

A. muciniphila
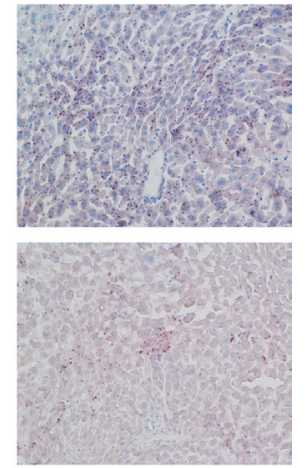

A. muciniphila

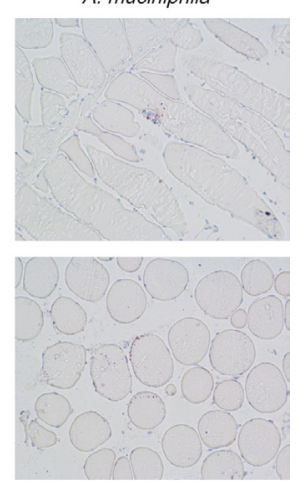

\section{Figure 2}

A. muciniphila supplementation reduced fat deposition in liver and muscle. (A) Relative mRNA levels of SREBP1C, ACC and CD36 in liver between A. muciniphila-supplemented mice and their PBS-treated counterparts. (B) Relative mRNA levels of SREBP1C, ACC and CD36 in muscle, as indicated in $B$. (C) Relative mRNA levels of SREBP1C, ACC and CD36 in epididymal adipose tissue ( $n=9-8$ for each group, data were shown as mean \pm S.D., unpaired two-tailed $t$-test, $* P<0.05$, $* * P<0.01$ ). ( $D$ and $E$ ) Representative Oil Red $O$ staining for fat deposition measurement in liver (D) and muscle (E) between A. muciniphilasupplemented mice and the control group.

\section{A. muciniphila supplementation attenuates ER stress in metabolic tissues}

Recent evidence recapitulates the interaction among fat deposition, inappropriate activation of ER stress and insulin resistance (Goodpaster et al. 2001, Ozcan et al. 2004). Therefore, we hypothesized that the improvement of glucose tolerance by $A$. muciniphila supplementation may be partly dependent on the reduced lipotoxicity and ER stress. We further measured the mRNA expression of markers related to ER stress, and in line with the changes in lipid accumulation in liver and muscle, we detected the downregulation of unfolded protein response signals representing ER stress such as immunoglobulin heavy chain-binding protein/glucose-regulated protein 78 (BiP/ GRP78) and PKR-like ER kinase (PERK) in the liver (Fig. 3A) in the A. muciniphila group. These proteins could facilitate diverse functions in the endoplasmic reticulum such as folding and assembly of newly synthesized proteins and initiation of unfolded protein response (Shi et al. 1998). The reduced expression levels of BiP, PERK protein disulfide isomerase (PDI) and XBP1 were also observed in the muscle of mice treated with A. muciniphila (Fig. 3B). However, similar changes were not detected in the adipose tissues (Fig. 3C). Protein levels of ER stress indicators such as phosphorylated EIF2A and phosphorylated PERK were assessed in both hepatic and muscle tissues. Consistent with the result of gene expression, levels of p-EIF2A and p-PERK were lower in liver and muscle of A. muciniphilatreated mice compared with those of controls (Fig. 3D and E). These results supported our hypothesis that the improvement of glucose tolerance by A. muciniphila supplementation is associated with the alleviation of ectopic intracellular lipid accumulation and ER stress.

\section{A. muciniphila supplementation improves chronic low-grade inflammation}

The interactions between inflammation and insulin resistance are widely accepted nowadays (Shoelson et al. 2006). Moreover, it is reported that A. muciniphila administration could restore gut barrier function, decrease intestinal permeability and may prevent the development of high-fat diet-induced metabolic endotoxemia, as revealed by decreased circulating LPS levels (Everard et al. 2013). Here, we detected a significantly reduced level of plasma LBP (Fig. 4A), which is the indicator of circulating LPS (Romani et al. 2013), in A. muciniphila-treated chow diet-fed mice. Meanwhile, inflammation factors such as leptin were also decreased by A. muciniphila (Fig. 4B).

Published by Bioscientifica Ltd 
A $\quad$ PBS

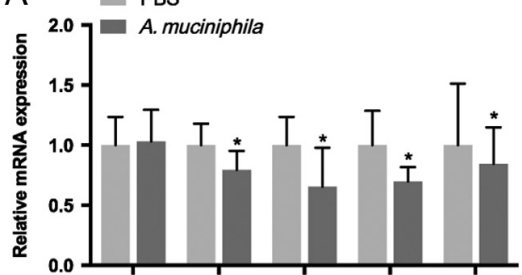

B

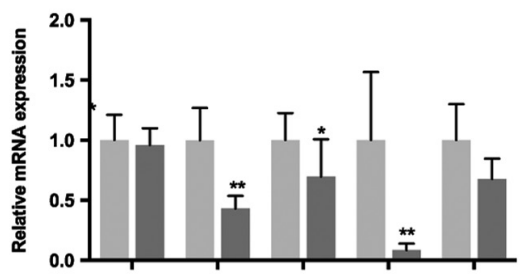

C

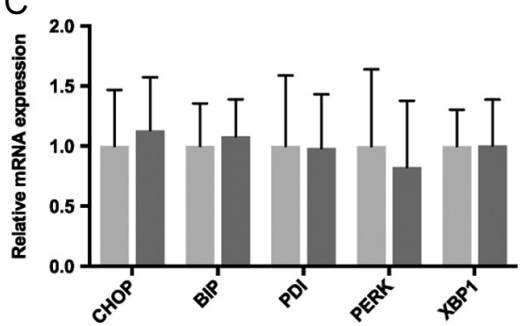

D
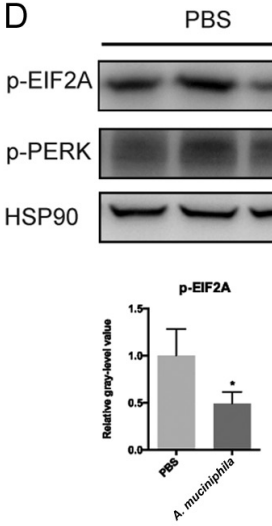

E

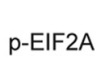

p-PERK

HSP90
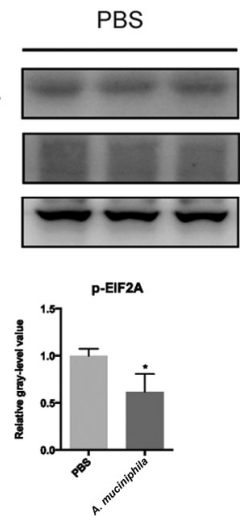
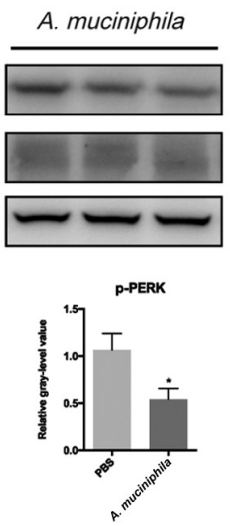

A. muciniphila
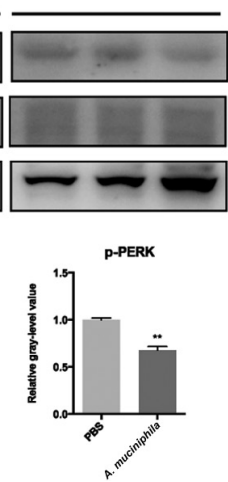

Figure 3

A. muciniphila supplementation influences the expression of ER stress-related genes in liver and muscle. (A) Real-time PCR results of CHOP, BIP, PDI, PERK and XBP1 in liver. (B) Real-time PCR results of $\mathrm{CHOP}, \mathrm{BIP}, \mathrm{PDI}, \mathrm{PERK}$ and $\mathrm{XBP} 1$ in muscle between $A$. muciniphila-administered group and PBS-treated vehicle. (C) Real-time PCR results of CHOP, BIP, PDI, PERK and XBP1 in epididymal adipose tissue $(n=9-8$ for each group, data were shown as mean \pm S.D., unpaired two-tailed $t$-test, $* P<0.05, * * P<0.01)$. ( $D$ and $E$ ) The levels of $p$-EIF2A and $p$-PERK involved in the unfolded protein response were detected both in hepatic tissue (D) and in muscle (E) of $A$. muciniphilasupplemented mice and their counterparts, as well as the quantitation of representative proteins. All these samples were from 3 different mice from each of the two groups.
It was evidenced that the attenuated ER stress of the intestine is associated with inflammation and the gut barrier function (Ma 2008). We also detected a decrease in the mRNA expression levels of ER stress-related genes, CHOP and TRIB3 in colon (Supplementary Fig. 3A) and a decrease in PDI mRNA levels in jejunum (Supplementary Fig. 3B). It is because CHOP, TRIB3 and PDI are ER stress transducers, leading to the activation of unfolded protein response (Cnop et al. 2012). These results suggested that A. muciniphila supplementation alleviated ER stress in the intestine and chronic endotoxemia.

LPS, known as an endotoxin, could enter the circulation through the impaired intestinal mucosa, and then bind to LBP, which can facilitate the interaction of LPS and its receptors, thereby triggering inflammatory cascades including nuclear factor-KB (NF-KB) and JNK activation (Zweigner et al. 2006, Tsaousidou et al. 2014). Correspondingly, we found that A. muciniphila supplementation significantly reduced phospho-JNK levels and increased the NF-KB inhibitor protein and IKBA protein levels in the liver (Fig. 4C), suggesting an inactivation of these two pathways in the A. muciniphila group. Similar changes were observed in muscle (Fig. 4D). These results demonstrated that A. muciniphila supplementation improved metabolic endotoxemia and the following local inflammation cascades, which might mediate the beneficial metabolic effects.

\section{Impact of $A$. muciniphila supplementation on the metabolomics profile of plasma}

Taken together, we demonstrated the beneficial effects of A. muciniphila administration on host metabolic profiles, metabolic endotoxemia and inflammation. In a recent study, it is well established that gut microbiota could ferment the non-digestible carbohydrates in the colon and produce metabolites that have profound effects on health (De Vadder et al. 2014). Therefore, we further investigated the metabolomics profile in the plasma of both the A. muciniphila-treated group and their counterparts to clarify whether $A$. muciniphila treatment resulted in a more favorable metabolomics profile (Table 1). Interestingly, among these metabolites, the levels of citrulline and ornithine were significantly lowered by $A$. muciniphila supplementation. Previous studies observed that these two amino acids were higher in obese and diabetic mice and also in humans with obesity (Mochida et al. 2011, Verdam et al. 2011, Sailer et al. 2013). These findings may indicate the possible involvement of these amino

Published by Bioscientifica Ltd 
A

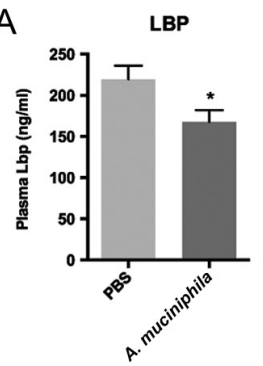

C

P-JNK

JNK
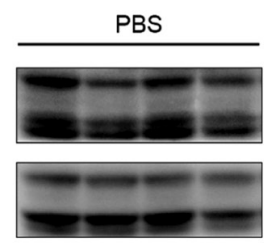

IKBA

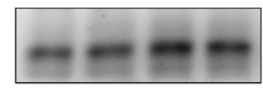

HSP9O

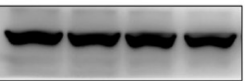

D

P-JNK

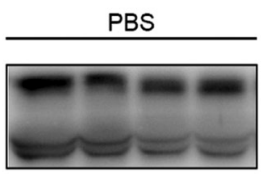

JNK

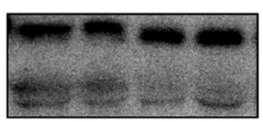

IKBA

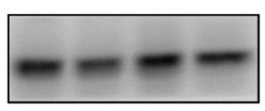

HSP9O

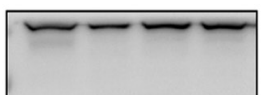

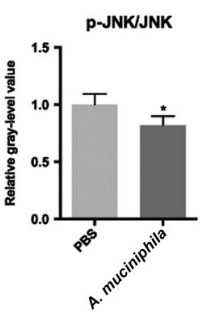
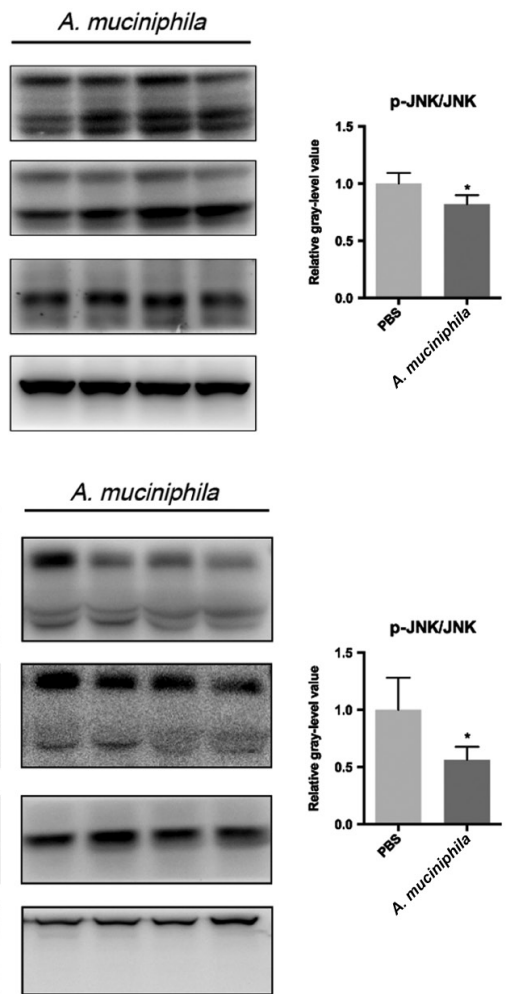

acids in metabolism. Besides, we detected an increase in A-tocopherol concentrations, which acted as an essential antioxidant and anti-inflammatory factor (Gren 2013), as well as an increased levels of B-sitosterol that boosts the immune system and exhibits an anti-inflammatory activity in endothelial cells including intestinal endothelial cells (Loizou et al. 2010). These results further support the antiinflammatory benefits by A. muciniphila supplementation.

\section{Discussion}

Growing evidence supports the crosstalk between gut microbiota and host health, and alterations in the composition of gut microbiota are involved in the development of metabolic disorders such as obesity and T2DM (Hartstra et al. 2015). Thus, elucidating the effects of candidate bacterial species on host metabolism will provide new methods for preventing and treating metabolic diseases. In this study, we examined the

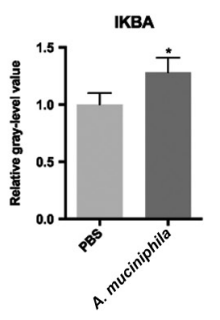

\section{Figure 4}

A. muciniphila supplementation is beneficial to improve the low-grade inflammation. (A) Plasma LBP levels ( $n=9-6$ for each group, data were shown as mean \pm S.D., unpaired two-tailed $t$-test, $* P<0.05, * * P<0.01)$. (B) Plasma leptin levels ( $n=8-6$ for each group, data were shown as mean \pm S.D., unpaired two-tailed $t$-test, $* P<0.05$, $* * P<0.01)$. (C and $D)$ Immunoblotting analysis with antibodies against phospho-JNK, total JNK, IKBA and HSP9O of liver (C) and muscle (D), the levels of phospho-Jnk were normalized to the levels of total Jnk, and other protein levels were normalized to Hsp90. Relative gray level value of these proteins were illustrated in figure (C) and (D).

metabolic benefits of $A$. muciniphila in mice fed chow diet. Interestingly, we found that $A$. muciniphila daily gavage for five weeks significantly improved glucose tolerance and insulin sensitivity in liver and muscle. Correspondingly, fat deposition and ER stress were alleviated in liver and muscle by A. muciniphila. More importantly, A. muciniphila supplementation reduced metabolic endotoxemia and inflammation signaling in liver and muscle. These results firstly identified the metabolic benefits of A. muciniphila in metabolic healthy mice, providing the possibilities to prevent the metabolic disturbances using probiotics in the general population.

The mucin-degrading bacterium A. muciniphila is established during the first month of human life and resides in the mucosa of healthy adults (Collado et al. 2007). Abundant evidence has demonstrated that the abundance of $A$. muciniphila is reduced in obese patients and mice (Everard et al. 2013, Le Chatelier et al. 2013),

Published by Bioscientifica Ltd. 
Table 1 Significantly different plasma metabolites between Akkermansia muciniphila-intervened mice and the control.

\begin{tabular}{|c|c|c|c|c|c|c|}
\hline No. & Metabolites $^{a}$ & $\mathbf{R T}(\min )$ & $\mathbf{m} / \mathbf{z}$ & $\begin{array}{l}\text { VIP valueb } \\
\text { (OPLS-DA) }\end{array}$ & $\begin{array}{c}\boldsymbol{P} \text { value } \\
\text { (Student's } t \text {-test) }\end{array}$ & ${\text { Fold-changed }\left(\log _{2}\right)}$ \\
\hline 1 & AMP (adenosine monophosphate) & 30 & 315 & 1.9 & 0 & -1 \\
\hline 2 & Hydrocaffeic acid & 23 & 398 & 1.7 & 0 & -0.6 \\
\hline 3 & 5-aminovaleric acid & 19 & 174 & 1.7 & 0 & -0.6 \\
\hline 4 & 2-ketoglutaric acid & 18 & 198 & 1.6 & 0 & -0.6 \\
\hline 5 & Pipecolinic acid & 14 & 156 & 2 & 0 & -0.3 \\
\hline 6 & Ornithine & 19 & 142 & 1.6 & 0 & -0.2 \\
\hline 7 & Citrulline & 22 & 157 & 1.9 & 0 & -0.2 \\
\hline 8 & Pyrophosphate & 19 & 451 & 1.7 & 0 & -0.1 \\
\hline 9 & Acetoacetate & 8.8 & 188 & 1.6 & 0 & 0.1 \\
\hline 10 & Malonic acid & 10 & 233 & 1.6 & 0 & 0.4 \\
\hline 11 & Fucose & 20 & 117 & 1.8 & 0 & 0.2 \\
\hline 12 & 4-hydroxyproline & 17 & 230 & 1.5 & 0 & 0.3 \\
\hline 13 & Uric acid & 25 & 441 & 2.1 & 0 & 0.4 \\
\hline 14 & 3,4-dihydroxybutyric acid & 15 & 233 & 2.2 & 0 & 0.4 \\
\hline 15 & $\alpha$-tocopherol & 31 & 502 & 2 & 0 & 0.9 \\
\hline 16 & $\beta$-sitosterol & 33 & 129 & 1.8 & 0 & 0.2 \\
\hline 17 & Cholesterol & 31 & 329 & 1.8 & 0 & 0.3 \\
\hline
\end{tabular}

aThe metabolites were structurally identified by reference standards. bVariable importance in the projection (VIP) was obtained from the OPLS-DA model. cThe $P$ value was calculated from Student's $t$-test. dFold-change was calculated as a binary logarithm of the average mass response (normalized peak area) ratio between $A$. muciniphila group vs PBS group, where a positive value means that the average mass response of the metabolite in the $A$. muciniphila group is larger than that in the PBS group.

whereas its abundance is increased in obese patients after weight loss treatment (Liou et al. 2013) or in diabetic mice after lowering glucose by metformin (Shin et al. 2014). There are also studies revealing the anti-diabetic effects of A. muciniphila in high-fat diet (HFD)-induced diabetic mice (Everard et al. 2013, Shin et al. 2014). We firstly observed that $A$. muciniphila supplementation significantly improved body weight gain, fat mass and glucose tolerance in chow diet-fed mice. The reduction in fat mass accompanied an increase in lean mass indicating a body composition change by A. muciniphila. Severely reduced fat mass (such as lipodystrophy) is related to insulin resistance (Shay et al. 2011). In our study, A. muciniphila supplementation improved glucose tolerance and insulin sensitivity. These results overall suggested that the decreased fat mass and increased lean mass by $A$. muciniphila may also metabolically benefit the relatively metabolic healthy subjects. We noticed that a previous study reported by Everard and coworkers did not find similar changes in chow diet-fed mice (Everard et al. 2013). The discrepancy might be due to the differences in the length of $A$. muciniphila treatment or the diet content. Both studies used C57BL/6 mice. However, we treated mice with A. muciniphila for five weeks in a chow diet containing $9.7 \%$ water, $20.5 \%$ protein, $4.62 \%$ fat, $4.35 \%$ fiber and $52.5 \%$ nitrogen-free extract, whereas Everard and coworkers treated mice for four weeks in a control diet (AIN93Mi, Research Diet) containing 6.8\% water,
$12.5 \%$ protein, $4 \%$ fat and $72.7 \%$ carbohydrate, suggesting that $A$. muciniphila's effect may be diet dependent. It is previously reported that $A$. muciniphila affected intestine length and energy absorption during cold (Chevalier et al. 2015). We found that $A$. muciniphila did not affect the intake or energy excretion from the gut at least in ambient temperature. The detailed mechanism of $A$. muciniphila in energy homeostasis remains to be clarified in future study.

Everard and coworkers have demonstrated that A. muciniphila treatment alleviated fat storage in adipose tissue in HFD-induced obese mice (Everard et al. 2013). In our study, we did not observe the changed expression of genes related to lipid synthesis or transport in fat tissues in the chow diet-fed A. muciniphila group compared with the control group, suggesting that $A$. muciniphila's effects on fat storage may be affected by diet status. However, interestingly, we found a significant reduction in the expression levels of genes involved in fatty acid synthesis and transport in non-fat insulin-responsive tissues such as liver and muscle in $A$. muciniphila-treated mice. The immunoblotting analysis with antibodies against phospho-JNK, total JNK, IKBA and HSP90 of liver and muscle and relative gray level value of these proteins were also illustrated, indicating a reduced lipid accumulation by $A$. muciniphila, which may be one reason for the elevated insulin signaling activation in liver and muscle in A. muciniphila group, as it is well established that lipid accumulation in liver and muscle triggers pathways

Published by Bioscientifica Ltd. 
that impair insulin signaling thereby insulin resistance (Samuel \& Shulman 2016).

We found that A. muciniphila daily gavage for 5 weeks significantly improved glucose tolerance and insulin sensitivity in liver and muscle. Key gluconeogenetic enzymes, PEPCK and G6PC (Lochhead et al. 2000), were decreased after A. muciniphila supplementation. However, we did not see a reduction in fasting blood glucose, consistent to previous study (Everard et al. 2013) indicating that other regulators such as liver glycogenolysis, gluconeogenesis in the kidneys or intestine (Mutel et al. 2011, Al-Gayyar et al. 2015) may also be involved in fasting glucose regulation by $A$. muciniphila.

ER plays a central role in the embellishment of lipid and protein. The protein synthesized in ER would fail to acquire correct decoration, and misfolded proteins would accumulate in ER under pathological conditions, causing ER stress and activating unfolded protein response signal (Ozcan et al. 2004). Murine studies have revealed that ER stress is one of the possible mechanisms responsible for triggering innate immune inflammatory pathways and insulin resistance (Hotamisligil 2010). Besides, ER membranes also contain key lipogenic enzymes affecting the biosynthesis of lipid, which further impair insulin signaling (Lee et al. 2008). Our work showed that ER stress in liver and muscle was significantly inhibited by
A. muciniphila treatment, in accordance with the reduced lipid accumulation and activated insulin signaling in these two organs.

We have demonstrated the beneficial effects of A. muciniphila supplementation on host metabolism. The next question is how these effects were achieved by oral administration of A. muciniphila. As A. muciniphila is well demonstrated to maintain the intestinal barrier (Everard et al. 2013), which is important to prevent the pathogenic microbial products from entering into circulation, whereas LPS, a known endotoxin, is a crucial component of gram-negative bacteria cell walls, which could enter the circulation through an impaired gut barrier. Recent studies demonstrate that a high-fat-containing diet alters the diversity of gut microbiota and intestinal wall permeability, elevating enterobacterial production and translocation of LPS into the systemic circulation, as referred to metabolic endotoxemia (Cani et al. 2007). In circulation, LBP takes up and binds to LPS with high affinity, facilitates LPS activation and transfers it to the TLR4 receptor complex, initiating downstream inflammation signaling that further influences the insulin sensitivity and glucose disposal (Wright et al. 1990). We detected that plasma LBP levels were significantly reduced by $A$. muciniphila treatment. As LBP is an indicator of circulating LPS, our result suggested that the reduced metabolic endotoxemia in

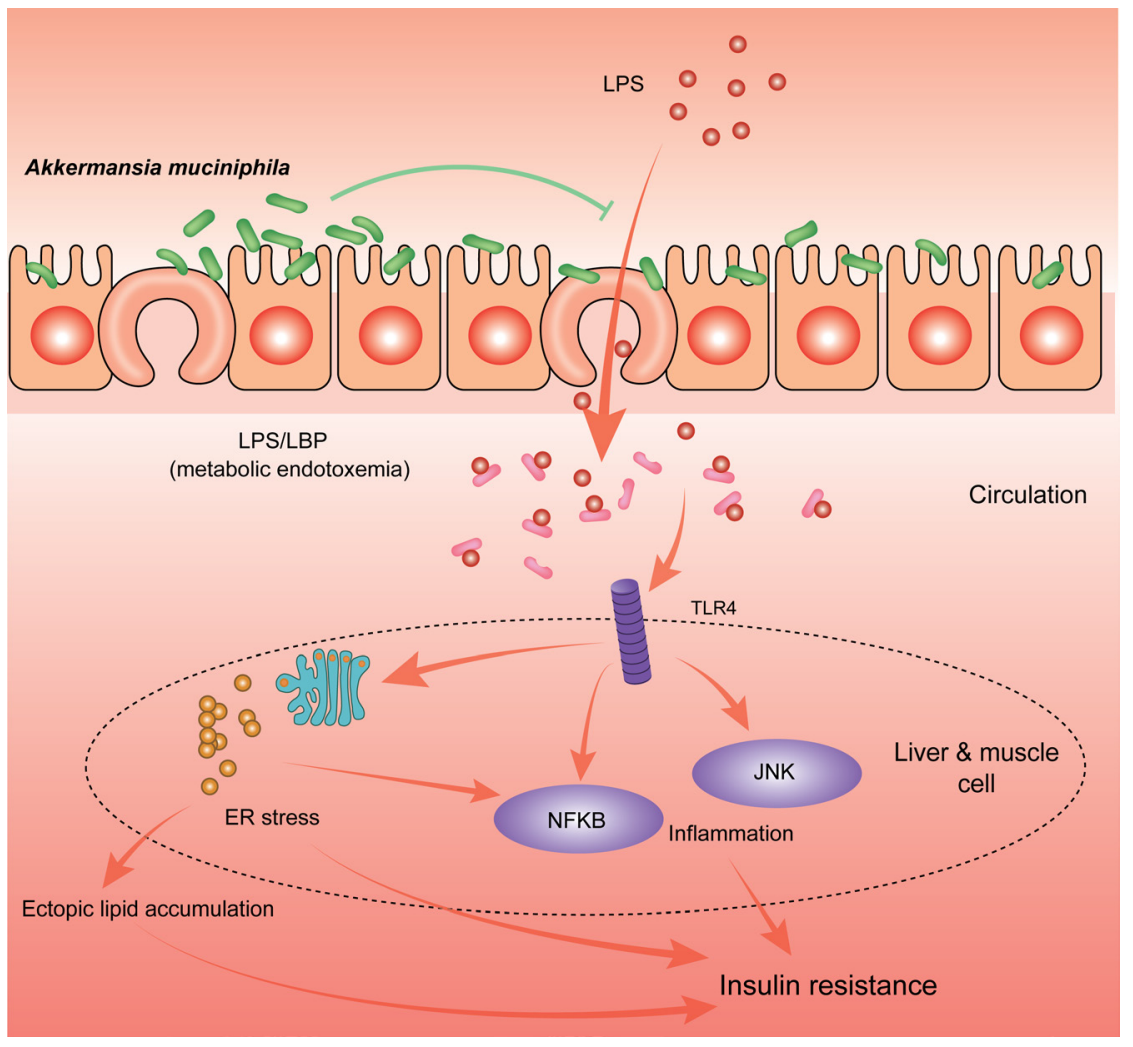

Figure 5

Graphical abstract. Functional interaction between A. muciniphila and host metabolism. A. muciniphila residing in the mucus layer of intestine and maintaining gut barrier inhibits bacteria-derived LPS penetrating into circulation (metabolic endotoxemia), thereby alleviating LPS/ LBP/TLR4-initiated inflammation cascade and ER stress in liver and muscle cells, and the inhibited ER stress further inactivated inflammatory pathways and fat deposition, which altogether led to activated insulin signaling in liver and muscle and improved glucose tolerance. $E R$, endoplasmic reticulum; LBP, LPS-binding protein; LPS, lipopolysaccharide; TLR4, toll-like receptor 4 . 
A. muciniphila-treated chow diet-fed mice. This effect mainly depends on the gut barrier maintenance ability of $A$. muciniphila (Everard et al. 2013). Furthermore, the LPS/LBP downstream signaling such as JNK and NF-KB (Wright et al. 1990) was also inhibited by $A$. muciniphila treatment. These results demonstrated the improved endotoxemia and chronic inflammation by $A$. muciniphila supplementation. It is well testified that JNK can bind to IRS1 and lead to Ser307 phosphorylation, resulting in a decrease of phosphorylated AKT and inactivation of insulin signaling (Hirosumi et al. 2002); and activated IKK-NF-KB pathway also plays a central role in the development of insulin resistance and metabolic abnormalities (Hirosumi et al. 2002). There is also evidence demonstrating that additional LPS could induce ER stress in insulin-responsive cells (Wolfe et al. 2013). Moreover, circulating LBP levels are significantly associated with insulin resistance and various inflammatory markers (Moreno-Navarrete et al. 2012). These findings suggested that A. muciniphila supplementation initially alleviated metabolic endotoxemia from the gut, thereby inhibiting inflammation cascade and ER stress, and the inhibited ER stress further inactivated inflammatory pathways (Ozcan et al. 2004), which altogether led to activated insulin signaling in liver and muscle and improved global glucose tolerance (Fig. 5).

To further examine the interactions between A. muciniphila consumption and metabolic profiles, plasma metabolomics analysis was performed as it has been used to predict the progress of T2DM in advance of onset in human beings (Wang et al. 2011). Our results showed A. muciniphila treatment decreased the circulating levels of citrulline and ornithine as well as increased the circulating levels of $\alpha$-tocopherol and $\beta$-sitosterol. Amino acids are emerging as a new class of effective molecules in the etiology of obesity and diabetes mellitus (Schwenk \& Haymond 1987, Newgard et al. 2009). Previous studies observed that these two amino acids were higher in obese and diabetic mice and also in humans with obesity (Mochida et al. 2011, Verdam et al. 2011, Sailer et al. 2013), which may predict the development of metabolic syndromes. Moreover, A. muciniphila supplementation increased circulating levels of $\alpha$-tocopherol and $\beta$-sitosterol which are reported to boost the immune system and reduce systemic low-grade inflammation (Bendich 1988, Fraile et al. 2012). Ample evidence has demonstrated that A-tocopherol can attenuate LPS-induced inflammatory cytokines secretion (Zhang et al. 2010, Lira et al. 2011). B-sitosterol also has beneficial effects directly on endothelial and monocytic cells (Bustos et al. 2008), alleviating HFD-induced intestinal inflammation by inhibiting the binding of LPS to toll-like receptor 4 in the NF-KB pathway (Kim et al. 2014). These results further supported the anti-inflammation effects and protective effects on the gut barrier function of A. muciniphila.

In summary, for the first time, we demonstrated that A. muciniphila supplementation in mice fed a chow diet decreased metabolic endotoxemia, resulting in a more favorable metabolomics profiles in circulation, as well as the inhibited pro-inflammatory pathways, ER stress and lipogenesis in insulin-responsive tissues, leading to improved insulin action and glucose tolerance. Our study illustrated the crosstalk between A. muciniphila and host metabolism, providing the possible mechanisms of A. muciniphila's metabolic benefits such as glucose tolerance improvement. These results added new evidence for the supplementation of probiotics to metabolic healthy population and also suggested a new strategy to prevent the development of metabolic diseases such as T2DM at an even earlier stage.

Supplementary data

This is linked to the online version of the paper at http://dx.doi.org/10.1530/ JME-16-0054.

Declaration of interest

The authors declare that there is no conflict of interest that could be perceived as prejudicing the impartiality of the research reported.

\section{Funding}

This work was supported by the National International Science Cooperation Foundation (grant number 2015DFA30560), National Nature Science Foundation of China (grant numbers 81270931, 81370949, 81370963, $81471060,81522011,81570757$ and 81570758) and Program of Shanghai Subject Chief Scientist (grant number XBR2013073).

\section{Author contribution statement}

$\mathrm{R} \mathrm{L}$ and $\mathrm{J} \mathrm{H}$ conceived the project and designed the experiments. S $\mathrm{Z}$ and W L carried out most of the experiments. J W, R L and S Z wrote the paper. Y S contributed with the mice breeding and animal experiments. J $S$ assisted with statistical analysis. W W and G N contributed comments and advice on the manuscript. All authors were involved in editing the manuscript.

\section{Acknowledgements}

The authors thank Peijun Yin, Shasha Wang and Hongjing Yu from Shanghai Xinyi Pharmaceutical Company for their assistance in cultivating bacteria.

\section{References}

Al-Gayyar MM, Alyoussef A, Hamdan AM, Abbas A, Darweish MM \& El-Hawwary AA 2015 Cod liver oil ameliorates sodium nitriteinduced insulin resistance and degradation of rat hepatic glycogen

Published by Bioscientifica Ltd 
through inhibition of cAMP/PKA pathway. Life Sciences 120 13-21. (doi:10.1016/j.lfs.2014.11.002)

Anhe FF, Roy D, Pilon G, Dudonne S, Matamoros S, Varin TV, Garofalo C, Moine Q, Desjardins Y, Levy E, et al. 2015 A polyphenolrich cranberry extract protects from diet-induced obesity, insulin resistance and intestinal inflammation in association with increased Akkermansia spp. population in the gut microbiota of mice. Gut $\mathbf{6 4}$ 872-883. (doi:10.1136/gutjnl-2014-307142)

Belzer C \& de Vos WM 2012 Microbes inside - from diversity to function: the case of Akkermansia. ISME Journal 6 1449-1458. (doi:10.1038/ismej.2012.6)

Bendich A 1988 Vitamin E and immune functions. Basic Life Sciences 49 615-620. (doi:10.1007/978-1-4684-5568-7 96)

Bustos P, Duffau C, Pacheco C \& Ulloa N 2008 beta-Sitosterol modulation of monocyte-endothelial cell interaction: a comparison to female hormones. Maturitas 60 202-208. (doi:10.1016/j.maturitas.2008.06.002)

Cani PD, Amar J, Iglesias MA, Poggi M, Knauf C, Bastelica D, Neyrinck AM, Fava F, Tuohy KM, Chabo C, et al. 2007 Metabolic endotoxemia initiates obesity and insulin resistance. Diabetes $\mathbf{5 6}$ 1761-1772. (doi:10.2337/db06-1491)

Cani PD, Possemiers S, Van de Wiele T, Guiot Y, Everard A, Rottier O, Geurts L, Naslain D, Neyrinck A, Lambert DM, et al. 2009 Changes in gut microbiota control inflammation in obese mice through a mechanism involving GLP-2-driven improvement of gut permeability. Gut 58 1091-1103. (doi:10.1136/gut.2008.165886)

Cani PD, Osto M, Geurts L \& Everard A 2012 Involvement of gut microbiota in the development of low-grade inflammation and type 2 diabetes associated with obesity. Gut Microbes 3 279-288. (doi:10.4161/gmic.19625)

Cano PG, Santacruz A, Trejo FM \& Sanz Y 2013 Bifidobacterium CECT 7765 improves metabolic and immunological alterations associated with obesity in high-fat diet-fed mice. Obesity 21 2310-2321. (doi:10.1002/oby.20330)

Capel F, Rolland-Valognes G, Dacquet C, Brun M, Lonchampt M, Ktorza A, Lockhart B \& Galizzi JP 2013 Analysis of sterol-regulatory element-binding protein 1c target genes in mouse liver during aging and high-fat diet. Journal of Nutrigenetics and Nutrigenomics $\mathbf{6}$ 107-122. (doi:10.1159/000350751)

Chevalier C, Stojanovic O, Colin DJ, Suarez-Zamorano N, Tarallo V, VeyratDurebex C, Rigo D, Fabbiano S, Stevanovic A, Hagemann S, et al. 2015 Gut microbiota orchestrates energy homeostasis during cold. Cell 163 1360-1374. (doi:10.1016/j.cell.2015.11.004)

Cnop M, Foufelle F \& Velloso LA 2012 Endoplasmic reticulum stress, obesity and diabetes. Trends in Molecular Medicine 18 59-68. (doi:10.1016/j.molmed.2011.07.010)

Collado MC, Derrien M, Isolauri E, de Vos WM \& Salminen S 2007 Intestinal integrity and Akkermansia muciniphila, a mucindegrading member of the intestinal microbiota present in infants, adults, and the elderly. Applied and Environmental Microbiology $\mathbf{7 3}$ 7767-7770. (doi:10.1128/AEM.01477-07)

De Vadder F, Kovatcheva-Datchary P, Goncalves D, Vinera J, Zitoun C, Duchampt A, Backhed F \& Mithieux G 2014 Microbiota-generated metabolites promote metabolic benefits via gut-brain neural circuits. Cell 156 84-96. (doi:10.1016/j.cell.2013.12.016)

Derrien M, Vaughan EE, Plugge CM \& de Vos WM 2004 Akkermansia muciniphila gen. nov., sp. nov., a human intestinal mucin-degrading bacterium. International Journal of Systematic and Evolutionary Microbiology 54 1469-1476. (doi:10.1099/ijs.0.02873-0)

Derrien M, Collado MC, Ben-Amor K, Salminen S \& de Vos WM 2008 The mucin degrader Akkermansia muciniphila is an abundant resident of the human intestinal tract. Applied and Environmental Microbiology 74 1646-1648. (doi:10.1128/AEM.01226-07)

Everard A, Belzer C, Geurts L, Ouwerkerk JP, Druart C, Bindels LB, Guiot Y, Derrien M, Muccioli GG, Delzenne NM, et al. 2013 Cross-talk between Akkermansia muciniphila and intestinal epithelium controls dietinduced obesity. PNAS 110 9066-9071. (doi:10.1073/pnas.1219451110)
Fraile L, Crisci E, Cordoba L, Navarro MA, Osada J \& Montoya M 2012 Immunomodulatory properties of beta-sitosterol in pig immune responses. International Immunopharmacology 13 316-321. (doi:10.1016/j.intimp.2012.04.017)

Gao X, Pujos-Guillot E \& Sebedio JL 2010 Development of a quantitative metabolomic approach to study clinical human fecal water metabolome based on trimethylsilylation derivatization and GC/MS analysis. Analytical Chemistry 82 6447-6456. (doi:10.1021/ac1006552)

Goodpaster BH, He J, Watkins S \& Kelley DE 2001 Skeletal muscle lipid content and insulin resistance: evidence for a paradox in endurancetrained athletes. Journal of Clinical Endocrinology and Metabolism $\mathbf{8 6}$ 5755-5761. (doi:10.1210/jcem.86.12.8075)

Gren A 2013 Effects of vitamin E, C and D supplementation on inflammation and oxidative stress in streptozotocin-induced diabetic mice. International Journal for Vitamin and Nutrition Research $\mathbf{8 3}$ 168-175. (doi:10.1024/0300-9831/a000156)

Hansen CH, Krych L, Nielsen DS, Vogensen FK, Hansen LH, Sorensen SJ, Buschard K \& Hansen AK 2012 Early life treatment with vancomycin propagates Akkermansia muciniphila and reduces diabetes incidence in the NOD mouse. Diabetologia 55 2285-2294. (doi:10.1007/s00125012-2564-7)

Hartstra AV, Bouter KE, Backhed F \& Nieuwdorp M 2015 Insights into the role of the microbiome in obesity and type 2 diabetes. Diabetes Care 38 159-165. (doi:10.2337/dc14-0769)

Hirosumi J, Tuncman G, Chang L, Gorgun CZ, Uysal KT, Maeda K, Karin M \& Hotamisligil GS 2002 A central role for JNK in obesity and insulin resistance. Nature 420 333-336. (doi:10.1038/nature01137)

Hossain P, Kawar B \& El Nahas M 2007 Obesity and diabetes in the developing world - a growing challenge. New England Journal of Medicine 356 213-215. (doi:10.1056/NEJMp068177)

Hotamisligil GS 2010 Endoplasmic reticulum stress and the inflammatory basis of metabolic disease. Cell 140 900-917. (doi:10.1016/j.cell.2010.02.034)

Kim KA, Lee IA, Gu W, Hyam SR \& Kim DH 2014 beta-Sitosterol attenuates high-fat diet-induced intestinal inflammation in mice by inhibiting the binding of lipopolysaccharide to toll-like receptor 4 in the NF-kappaB pathway. Molecular Nutrition and Food Research $\mathbf{5 8}$ 963-972. (doi:10.1002/mnfr.201300433)

Kim MJ, Kim JE, Lee W \& Park SY 2015 Sfrp2 is a transcriptional target of SREBP-1 in mouse chondrogenic cells. Molecular and Cellular Biochemistry 406 163-171. (doi:10.1007/s11010-015-2434-y)

Le Chatelier E, Nielsen T, Qin J, Prifti E, Hildebrand F, Falony G, Almeida M, Arumugam M, Batto JM, Kennedy S, et al. 2013 Richness of human gut microbiome correlates with metabolic markers. Nature 500 541-546. (doi:10.1038/nature12506)

Lee AH, Scapa EF, Cohen DE \& Glimcher LH 2008 Regulation of hepatic lipogenesis by the transcription factor XBP1. Science 320 1492-1496. (doi:10.1126/science.1158042)

Lefterova MI, Zhang Y, Steger DJ, Schupp M, Schug J, Cristancho A, Feng D, Zhuo D, Stoeckert CJ Jr, Liu XS, et al. 2008 PPARgamma and C/EBP factors orchestrate adipocyte biology via adjacent binding on a genome-wide scale. Genes and Development 22 2941-2952. (doi:10.1101/gad.1709008)

Liou AP, Paziuk M, Luevano JM Jr, Machineni S, Turnbaugh PJ \& Kaplan LM 2013 Conserved shifts in the gut microbiota due to gastric bypass reduce host weight and adiposity. Science Translational Medicine 5 178ra141. (doi:10.1126/scitranslmed.3005687)

Lira FS, Rosa JC, Cunha CA, Ribeiro EB, do Nascimento CO, Oyama LM \& Mota JF 2011 Supplementing alpha-tocopherol (vitamin E) and vitamin D3 in high fat diet decrease IL-6 production in murine epididymal adipose tissue and 3T3-L1 adipocytes following LPS stimulation. Lipids in Health and Disease 10 37. (doi:10.1186/1476-511X-10-37)

Lochhead PA, Salt IP, Walker KS, Hardie DG \& Sutherland C 2000 5-aminoimidazole-4-carboxamide riboside mimics the effects of insulin on the expression of the 2 key gluconeogenic genes PEPCK and glucose6-phosphatase. Diabetes 49 896-903. (doi:10.2337/diabetes.49.6.896) 
Loizou S, Lekakis I, Chrousos GP \& Moutsatsou P 2010 Beta-sitosterol exhibits anti-inflammatory activity in human aortic endothelial cells. Molecular Nutrition and Food Research 54 551-558. (doi:10.1002/ mnfr.200900012)

Ma A 2008 Unresolved ER stress inflames the intestine. Cell 134 724-725. (doi:10.1016/j.cell.2008.08.023)

Mochida T, Tanaka T, Shiraki Y, Tajiri H, Matsumoto S, Shimbo K, Ando T, Nakamura K, Okamoto M \& Endo F 2011 Time-dependent changes in the plasma amino acid concentration in diabetes mellitus. Molecular Genetics and Metabolism 103 406-409. (doi:10.1016/j.ymgme.2011.05.002)

Moreno-Navarrete JM, Ortega F, Serino M, Luche E, Waget A, Pardo G, Salvador J, Ricart W, Fruhbeck G, Burcelin R, et al. 2012 Circulating lipopolysaccharide-binding protein (LBP) as a marker of obesityrelated insulin resistance. International Journal of Obesity 36 1442-1449. (doi:10.1038/ijo.2011.256)

Mutel E, Gautier-Stein A, Abdul-Wahed A, Amigo-Correig M, Zitoun C, Stefanutti A, Houberdon I, Tourette JA, Mithieux G \& Rajas F 2011 Control of blood glucose in the absence of hepatic glucose production during prolonged fasting in mice: induction of renal and intestinal gluconeogenesis by glucagon. Diabetes $603121-3131$. (doi:10.2337/db11-0571)

Newgard CB, An J, Bain JR, Muehlbauer MJ, Stevens RD, Lien LF, Haqq AM, Shah SH, Arlotto M, Slentz CA, et al. 2009 A branchedchain amino acid-related metabolic signature that differentiates obese and lean humans and contributes to insulin resistance. Cell Metabolism 9 311-326. (doi:10.1016/j.cmet.2009.02.002)

Ozcan U, Cao Q, Yilmaz E, Lee AH, Iwakoshi NN, Ozdelen E, Tuncman G, Gorgun C, Glimcher LH \& Hotamisligil GS 2004 Endoplasmic reticulum stress links obesity, insulin action, and type 2 diabetes. Science 306 457-461. (doi:10.1126/ science.1103160)

Qin J, Li Y, Cai Z, Li S, Zhu J, Zhang F, Liang S, Zhang W, Guan Y, Shen D, et al. 2012 A metagenome-wide association study of gut microbiota in type 2 diabetes. Nature 490 55-60. (doi:10.1038/ nature11450)

Reunanen J, Kainulainen V, Huuskonen L, Ottman N, Belzer C, Huhtinen H, de Vos WM \& Satokari R 2015 Akkermansia muciniphila adheres to enterocytes and strengthens the integrity of the epithelial cell layer. Applied and Environmental Microbiology $\mathbf{8 1}$ 3655-3662. (doi:10.1128/AEM.04050-14)

Romani J, Caixas A, Escote X, Carrascosa JM, Ribera M, Rigla M, Vendrell J \& Luelmo J 2013 Lipopolysaccharide-binding protein is increased in patients with psoriasis with metabolic syndrome, and correlates with C-reactive protein. Clinical and Experimental Dermatology 38 81-84. (doi:10.1111/ced.12007)

Sailer M, Dahlhoff C, Giesbertz P, Eidens MK, de Wit N, Rubio-Aliaga I, Boekschoten MV, Muller M \& Daniel H 2013 Increased plasma citrulline in mice marks diet-induced obesity and may predict the development of the metabolic syndrome. PLOS ONE 8 e63950. (doi:10.1371/journal.pone.0063950)

Samuel VT \& Shulman GI 2016 The pathogenesis of insulin resistance: integrating signaling pathways and substrate flux. Journal of Clinical Investigation 126 12-22. (doi:10.1172/JCI77812)

Schwenk WF \& Haymond MW 1987 Decreased uptake of glucose by human forearm during infusion of leucine, isoleucine, or threonine. Diabetes 36 199-204. (doi:10.2337/diab.36.2.199)

Shay CM, Carnethon MR, Church TR, Hankinson AL, Chan C, Jacobs DR Jr, Lewis CE, Schreiner PJ, Sternfeld B \& Sidney S 2011
Lower extremity fat mass is associated with insulin resistance in overweight and obese individuals: the CARDIA study. Obesity 19 2248-2253. (doi:10.1038/oby.2011.113)

Shi Y, Vattem KM, Sood R, An J, Liang J, Stramm L \& Wek RC 1998 Identification and characterization of pancreatic eukaryotic initiation factor 2 alpha-subunit kinase, PEK, involved in translational control. Molecular and Cellular Biology 18 7499-7509. (doi:10.1128/ MCB.18.12.7499)

Shin NR, Lee JC, Lee HY, Kim MS, Whon TW, Lee MS \& Bae JW 2014 An increase in the Akkermansia spp. population induced by metformin treatment improves glucose homeostasis in diet-induced obese mice. Gut 63 727-735. (doi:10.1136/gutjnl-2012-303839)

Shoelson SE, Lee J \& Goldfine AB 2006 Inflammation and insulin resistance. Journal of Clinical Investigation 116 1793-1801. (doi:10.1172/JCI29069)

Tong L 2005 Acetyl-coenzyme A carboxylase: crucial metabolic enzyme and attractive target for drug discovery. Cellular and Molecular Life Sciences 62 1784-1803. (doi:10.1007/s00018-005-5121-4)

Tsaousidou E, Paeger L, Belgardt BF, Pal M, Wunderlich CM, Bronneke H, Collienne U, Hampel B, Wunderlich FT, Schmidt-Supprian M, et al. 2014 Distinct roles for JNK and IKK activation in agouti-related peptide neurons in the development of obesity and insulin resistance. Cell Reports 9 1495-1506. (doi:10.1016/j.celrep.2014.10.045)

Ussar S, Griffin NW, Bezy O, Fujisaka S, Vienberg S, Softic S, Deng L, Bry L, Gordon JI \& Kahn CR 2015 Interactions between gut microbiota, host genetics and diet modulate the predisposition to obesity and metabolic syndrome. Cell Metabolism 22 516-530. (doi:10.1016/j.cmet.2015.07.007)

Verdam FJ, Greve JW, Roosta S, van Eijk H, Bouvy N, Buurman WA \& Rensen SS 2011 Small intestinal alterations in severely obese hyperglycemic subjects. Journal of Clinical Endocrinology and Metabolism 96 E379-E383. (doi:10.1210/jc.2010-1333)

Wang TJ, Larson MG, Vasan RS, Cheng S, Rhee EP, McCabe E, Lewis GD, Fox CS, Jacques PF, Fernandez C, et al. 2011 Metabolite profiles and the risk of developing diabetes. Nature Medicine 17 448-453. (doi:10.1038/nm.2307)

Wang J, Liu R, Wang F, Hong J, Li X, Chen M, Ke Y, Zhang X, Ma Q, Wang R, et al. 2013 Ablation of LGR4 promotes energy expenditure by driving white-to-brown fat switch. Nature Cell Biology $\mathbf{1 5}$ 1455-1463. (doi:10.1038/ncb2867)

Wilson CG, Tran JL, Erion DM, Vera NB, Febbraio M \& Weiss EJ 2016 Hepatocyte-specific disruption of CD36 attenuates fatty liver and improves insulin sensitivity in HFD-fed mice. Endocrinology 157 570-585. (doi:10.1210/en.2015-1866)

Wolfe A, Liang H, Hussey SE, Sanchez-Avila A, Tantiwong P \& Musi N 2013 Effect of lipopolysaccharide on inflammation and insulin action in human muscle. PLOS ONE 8 e63983. (doi:10.1371/journal. pone.0063983)

Wright SD, Ramos RA, Tobias PS, Ulevitch RJ \& Mathison JC 1990 CD14, a receptor for complexes of lipopolysaccharide (LPS) and LPS binding protein. Science 249 1431-1433. (doi:10.1126/science.1698311)

Zhang X, Zhong X, Zhou Y, Wang G, Du H \& Wang T 2010 Dietary RRRalpha-tocopherol succinate attenuates lipopolysaccharide-induced inflammatory cytokines secretion in broiler chicks. British Journal of Nutrition 104 1796-1805. (doi:10.1017/S0007114510002801)

Zweigner J, Schumann RR \& Weber JR 2006 The role of lipopolysaccharide-binding protein in modulating the innate immune response. Microbes and Infection 8 946-952. (doi:10.1016/j. micinf.2005.10.006)

Received in final form 20 October 2016 Accepted 6 November 2016

Accepted Preprint published online 7 November 2016 http://jme.endocrinology-journals.org

DOI: 10.1530/JME-16-0054
๑) 2017 Society for Endocrinology Printed in Great Britain 